\title{
DIAGNÓSTICO DE GESTAÇÃO POR ULTRA-SONOGRAFIA
}

\section{PREGNANCY DIAGNOSIS BY ULTRASOUND SCANNING}

\author{
- REVISÃO BIBLIOGRÁFICA -
}

\section{RESUMO}

Apresentam-se as vantagens, os princípios fundamentais da técnica e a situação atual do diagnóstico de gestação por ultra-sonografia nas espécies bovina, equiina e ovina. Entre as vantagens da ultra-sonografia para o diagnóstico de gestação destacase a precocidade, a inocuidade, o diagnóstico de prenhez gemelar e a possibilidade de estimar a viabilidade embrionária. O diagnóstico de gestação por ultra-sonografia possibilita um reconhecimento precoce e seguro desse estado com reflexos econômicos positivos e imediatos.

Palavras-chave: diagnóstico de gestação, ecografia, vaca, égua, ovelha.

\section{SUMMARY}

The advantagens, the principles, the technic and the updated status of pregnancy diagnosis in the cow, mare and ewe, by ultrasound scanning are presented in this paper. The early diagnosis twin pregnancies, evaluation of embryo viability and inocuity are some of the advantages of ultrasonography in domestic species.

Pregnancy diagnosis through ultrasound scanning allows safe early recognition of this state with imediate positive ecnonomic benefits.

\footnotetext{
* Médico Veterinário, Professor Titular, Doutor, Departamento de Clinica de Grandes Animais, Centro de Ciências Rurais, Universidade Federal de Santa Maria, 97.119 - Santa Maria, RS.
} 


\section{INTRODUÇÃO}

O conhecimento da existência ou não de gestação num animal é de considerável valor econômico. Em geral o diagnóstico precoce realizado logo após a cobertura ou inseminação permite identificar os animais não prenhes para tratamento imediato, se necessário, ou novo serviço, reduzindo ao mínimo a perda de tempo. É também importante para fins de seguro ou comercialização de animais, redução de perdas em programas que se utilizam de técnicas hormonais dispendiosas e sobretudo propicia um manejo mais adequado (HAFEZ, 1987).

o estabelecimento de uma gestação ocorre quando o concepto inibe a regressão do corpo lúteo prevenindo o retorno da fêmea ao estro. De modo geral, um animal que não retorna ao estro, após o serviço é considerado como prenhe. Este conhecimento é usualmente utilizado pelos criadores e centros de inseminação como indicativo de gestação. No entanto a confiança do método depende de precisão na detecção do estro, no rebanho, além do que tanto o anestro como o estro durante a gestação afetam a confiabilidade do método (HAFEZ, 1987).

Fundamentalmente a produção de imagens ecográficas ocorrem pela emissão de ondas ultra-sonoras pelos cristais, consequiente reflexão pelos tecidos e transformação deste eco em sinais luminosos no vídeo. Basicamente o aparelho consiste num transmissor de som ligado a um transdutor de
Cristais piezoelétricos que emitem o ultra-som que penetra nos tecidos; o eco refletido pelas barreiras das diferentes estruturas é captado pelo mesmo transdutor, conduzido até um receptor e levado a um amplificador de sinais elétricos que serão transmitidos a um tubo de raios catódicos com a definição de imagem numa tela. O exame assim feito é extremamente útil para avaliar os contornos, densidade e consistênCia dos órgãos (PALMER \& DRIANCOURT, 1980; GRIFFITH， 1980).

A ultra-sonografia é um método de diagnóstico que permite detectar a prenhez precoce, avaliar a idade gestacional, certas anormalidades que eventualmente possam ocorrer durante uma gestação como o desenvolvimento e anatomia fetais, além da gestação gemelar (TOTEY et al, 1991).

A determinação da gestação em estágios precoces permite não só a detecção de problemas associados à mortalidade embrionária como também sua detecção em tempo inferior ou correspondente $a$ de um ciclo estral, bem antes do diagnóstico realizado usualmente pela palpação retal ou mesmo dosagem do níveis de progesterona (PIERSON \& GINTHER, 1988).

GINTHER (1986) descreveu o ultra-som real-time como $O$ mais profundo avanço tecnológico no campo de estudo clínico e reprodutivo dos grandes animais, desde a introdução do radioimunoensaio, por ser um método de visualização não invasivo.

\section{ULTRA-SONOGRAFIA NAS DIFERENTES ESPÉCIES}




\section{BOVINOS}

Clinicamente, através da palpação retal, só é possível realizar o diagnóstico de gestação a partir dos 35 dias (GRUNERT \& BERCHTOLD, 1982).

CURRAN et al (1986a) detectaram a gestação de primíparas, pela imagem ultra-sônica, transretalmente, a partir do dia 11 pela visualização da vesícula embrionária até a percepçào do concepto no dia 20. As vesículas embrionárias ocuparam integralmente o corno ipsis-lateral ao corpo lúteo, em média, no dia 16 , e todo o contra-lateral a partir do dia 19. Durante o alongamento, as vesículas permaneceram com uma expessura aproximada de $2 \mathrm{~mm}$, e desenvolveram uma protuberância em todos os animais examinados. $\mathrm{Na}$ opinião dos mesmos autores o diagnóstico de gestação já pode ser realizado, através da ultrasonografia, pela detecção da vesícula embrionária esférica $(2,8 \mathrm{~mm})$ ou alorigada $(2,0 \quad x$ $4,5 \mathrm{~mm}), 12$ dias após a concepção com predomínio da forma esférica (73\%) sobre a alongada (27\%). 0 concepto pode ser detectado aos 20 dias, já com batimentos cardíacos, numa freqüência de 188 por minuto.

Utilizando um transdutor de $7,5 \mathrm{MHz}$, BOYD et al (1988) conseguiram detectar o concepto já aos 13 dias.

Em vacas Bos indicus, a vesícula embrionária pode ser visualizada entre os 18 e 20 dias pós-concepção, o concepto aos 19 , e os batimentos cardíacos aos 22 dias (TOTEY et al, 1991).
A detecção da prenhez em torno do dia 22 pode ser confundida com a presença de fluídos fisiológicos existentes no lúmen uterino, nas fases do proestro, estro e metaestro, embora a consistência uterina possa indicar a existência de uma gestação (FISSORE et al, 1986; KASTELIC, et al, 1991). Com estas considerações FISSORE et al (1986) conseguiram detectar o concepto somente aos 27 dias, pós-cobertura, pela observação de uma estrutura ecogênica no fluído não ecogênico.

A eficiência do diàgnóstico precoce de gestação foi avaliada por KASTELIC et al (1989) em novilhas holandesas utilizando dois examinadores, nos dias 10,12 , $14,16,18,20$ e 22 pós-inseminação. A precisão do diagnóstico foi de $50 \%$ até o dia 18 e de $100 \%$ nos dias 20 e 22 . Foi observado também a área luteal a qual permaneceu constante, neste período, nas gestantes, mas reduzida nas não prenhes. Também foi utilizado como parâmetro a textura uterina. Afirmaram ainda que considerando a reduzida dimensão do concepto, a precisão do exame depende da capacidade resolutiva do transdutor. Posteriormente (KASTELIC et al, 1991) concluíram que a capacidade de detecção da prenhez antes do dia 16 não é maior que 50\%, mesmo utilizando um transdutor de $7,5 \mathrm{MHz}$. Na opinião de PIETERSE et al (1990) e CHAFFAUX et al (1988) a detecção da prenhez entre os dias 21 e 25 é significativamente menos precisa que entre os dias 26 e 33 considerando a dificuldade de observação de pouca quanti- 
dade de líquido alantoidiano nos primeiros dias. Podem ainda ocorrer falsos positivos pela leitura de ecos provenientes da extremidade caudal do rúmen, bexiga repleta, cistos e coleções de líquidos na luz uterina.

A aparência do embrião apresenta variações segundo CURRAN et al (1986b). Aos 20 dias observaram uma linha, aos 25 dias uma forma de $\mathbf{C}$ e aos 33 uma forma de $L$.

Após os 20 dias outras estruturas tornam-se evidentes, não só facilitando o diagnóstico como permitindo uma estimativa da idade gestacional, tais como: alantóides, no dia 23; cordão espinhal, membros anteriores e âmnio no dia 29; área ótica, contorno do concepto e suas cavidades no dia 33; placentomas no dia 35; espaço interdigital e movimentos fetais no dia 44; costelas no dia 50 (CURRAN et al, 1986b; BOYD et al, 1988). Nas vacas Bos indicus, os parâmetros diferiram parcialmente. Assim os membros só foram detectados no dia 32 , o cordão espinhal no dia 33 e as costelas aos 60 dias (TOTEY et al, 1991).

\section{EQUIINOS}

O diagnóstico de gestação, na égua, pode ser feito, clinicamente, pela palpação das modificações uterinas, no início do desenvolvimento, apresentando muita precisão quando realizado após 25 dias da cobertura, conforme SIMPSON et al (1982). No entanto, com este método, é muito difícil ser detectada a vesícula embrionária com mais precocidade ou mesmo di- ferenciar quando forem gêmeas e estiveram no mesmo corno uterino (CHEVALIER \& PALMER， 1982; SIMPSON et al, 1982; PIPERS et al, 1984 ).

A precocidade no diagnóstico contribui significativamente para o manejo das éguas que apresentam vesículas gêmeas, das que estão vazias, ou com morte embrionária precoce, para que, caso necessário, sejam tomados procedimentos adequados antes da formação dos cálices endometriais (CHEVALIER \& PALMER， 1982; SIMPSON et al, 1982).

O diagnóstico de gestação através da ultra-sonografia já pode ser realizado pela identificação de uma vesícula de 10 a $15 \mathrm{~mm}$ de diâmetro, próximo a base de um dos cornos uterinos, em torno de 14 dias pós-ovulação, cuja forma é arredondada, com líquido no seu interior, aparecendo em preto no vídeo. A forma esférica do blastocisto, característica desta espécie, possibilita sua fácil identificação no interior do lúmen uterino, quando atingem um determinado diâmetro (PALMER \& DRIANCOURT, 1980).

Durante o início da gestação, a vesícula embrionária situa-se próximo a bifurcação dos corpos uterinos ou, então, na região cranial do cervix uterino (PALMER \& DRIANCOURT, 1980; SIMPSON et al, 1982). A identificação da vesícula embrionária pode ser facilitada pela visibilização de uma faixa de intensa ecogenicidade imediatamente abaixo do blastocisto e por um traço branco no seu polo dorsal. Isto se constitui num parâmetro seguro para 0 
diagnóstico porque corresponde a um artefato acústico que aparece frequientemente quando as ondas sonoras atravessam uma estrutura que contém líquido no seu interior (PIPERS et al, 1984).

A vesícula apresenta mobilidade aos 9-10 dias de gestação a qual aumenta até uma atividade máxima entre 11 e 15 dias, reduzindo entre os dias 15 e 16. Após o dia 17 não se observa mais movimento do concepto (GINTHER, 1983) .

o concepto eqüino apresenta aumento acentuado do seu diâmetro a partir do dia 14 pós-ovulação até próximo ao dia 29, variando de 14 a $16 \mathrm{~mm}$ entre o dia 15 e o dia 20. A partir daí as vesículas embrionárias permanecem com um diâmetro médio de $27 \mathrm{~mm}$ por 10 dias, aproximadamente (PALMER \& DRIANCOURT, 1980; SIMPSON et al, 1982; CHEVALIER \& PALMER, 1982; CORTE, 1990).

Entre os dias 20 a 28 ocorre uma pequena variação na forma da vesícula embrionária (PALMER \& DRIANCOURT, 1980) e entre os dias 25 e 29 é possível identificar dentro da vesícula uma formação ecogênica geralmente no polo ventral, representando 0 embrião (SIMPSON et al, 1982). Neste ponto, a medida que a vesícula cresce em diâmetro, o embrião se move em direção central. Entre os dias 30 e 39 de gestação, a imagem da vesícula embrionária retorna a forma ligeiramente circular medindo 25 a $40 \mathrm{~mm}$ de diâmetro (SIMPSON et al, 1982). Após 39 dias de idade o embrião apresenta-se suspenso no polo dorsal da vesícula e, em torno dos 50 dias,
- concepto pode ser visualizado na porção ventral da vesícula embrionária (PIPERS et al, 1984). Na opinião de CORTE (1990), esta modificação da forma se deve, provavelmente, ao aumento do tonus uterino, propiciando um crescimento da vesícula mais no sentido longitudinal.

As raças de cavalos de traÇão apresentam, aparentemente, vesículas embrionárias maiores do que as vistas nas raças leves, provavelmente, devido ao tamanho do útero. As éguas virgens tendem a apresentar vesículas embrionárias menores do que aquelas mais velhas e multíparas, talvez por apresentarem um trato genital menor (CHEVALIER \& PALMER, 1982).

Considerando o diâmetro das vesículas embrionárias no período de 19 a 28 dias de gestação, onde permanece em $27 \mathrm{~mm}$ de diâmetro, aproximadamente, verificado nos estudos de PALMER \& DRIANCOURT (1980), CHEVALIER \& PALMER (1982) e SIMPSON et al (1982) e ainda no estudo de PIPERS et al (1984), verifica-se que este dado não constitui um parâmetro seguro para avaliação do desenvolvimento da gestação, neste período. Os indicadores morfológicos tais Como a alteração na forma circular, visualização do embrião e do desenvolvimento das membranas alantoidianas devem ser observados para o acompanhamento seguro da gestação (GINTHER, 1986) .

CORTE (1990), confirmou a possibilidade de realização de um diagnóstico precoce de gestação já aos 14 dias. A mobilidade do concepto, sua evolução e o acompanhamento das características 
morfológicas do membranas, assim Ção embrionária embrião e suas como a reabsorem éguas puro sangue de corrida, também foram aspectos estudados pelo autor. Observou que durante os primeiros dias de gestação o concepto pode desaparecer e aparecer rapidamente no vídeo mesmo com um mínimo deslocamento do transdutor devido ao seu reduzido diâmetro e intensa mobilidade. Destacou que a detecção da reabsorção embrionária é feita pela constatação da redução do diâmetro das vesículas embrionárias, presença de contornos irregulares e ausência do em-
brião.

\section{OVINOS}

A utilização de machos vazectomizados ou capões androgenizados constitui-se num método simples e com custo inexpressivo para determinação do diagnóstico de gestação, mas apresenta limitações, pois simplesmente avalia a manifestação estral (WATT et al, 1984).

A impossibilidade de um diagnóstico de gestação, no primeiro terço gestacional justifica a importância da utilização da ecografia nesta espécie.

O diagnóstico precoce de gestação é de considerável valor econômico para o manejo racional das ovelhas, pois as não prenhes devem ser vendidas reduzindo os custos com a alimentação. As borregas vazias devem, igualmente, serem comercializadas enquanto alcançam bom preço, pois como adultas têm seu valor reduzido. Uma diferenciação entre prenhez simples e gemelar possibilita a elaboração de programa nutricional adequado na segunda metade da gestação (GEARHART et al, 1988).

A crescente utilização de programas de indução e sincronização estrais como utilização de técnicas hormonais na pré-estação reprodutiva requerem um diagnóstico precoce principalmente porque o não retorno ao cio também ocorrerá, neste caso, nas que não ficaram prenhes.

$\mathrm{Na}$ ovelha, o diagnóstico de gestação já pode ser realizado aos 30 dias através da utilização de um transdutor de $3 \mathrm{MHz}$ ou 5 $\mathrm{MHz}$, na região inguinal, em oveIhas em estação, pela visualização da vesícula embrionária, concepto, batimentos cardíacos e âmnio. Entretanto o período gestacional de 45 - 50 dias é o mais indicado para o diagnóstico (BUCKRELL, 1988; ALVES et al, 1991a). ALVES et al (1991a) avaliaram os procedimentos técnicos de utilização e definiram parâmetros para o diagnóstico de gestação em ovinos utilizando um transdutor linear de $2,4 \mathrm{MHz}$. O Exame realizado com a ovelha em estação, acesso do operador pela região posterior e posicionamento do transdutor na região pré-pública direita e esquerda se necessário, proporcionou maior facilidade para o diagnóstico. Consideraram como parâmetros das idades gestacionais o diâmetro do saco gestacional, comprimento cabeça-anca, o torácico, o cardíaco e a posição do útero na cavidade abdominal.

SERGEEV et al (1990) concluíram que as medidas, largura 
de cabeça e profundidade torácica são parâmetros aceitáveis para estimativa da idade fetal nas espécie ovina.

BUCKRELL (1986), utilizou a via retal para realização do diagnóstico de gestação nos dias 20-23 e aos 26-28, utilizando um transdutor de $7 \mathrm{Mhz}$ ou $5 \mathrm{MHz}$, considerando que nesta fase o útero ainda se encontra na cavidade pelvina. A manipulação do transdutor nesta espécie foi dificultada pelas dimensões do reto e por isso muitas das ovelhas reagiram por ocasião do exame. Por isso não recomendam a utilização da mesma, como rotina, com o equipamento que se dispõe atualmente. Neste sentido, WILKINS et al (1984), preconizam a não realização do diagnóstico antes dos 30 dias pela exigência de uma confirmação posterior, tendo em vista que a maior incidência de mortalidade embrionária é exatamente neste período. WHITE \& RUSSEL (1991) não recomendam qualquer atividade que envolva manejo antes dos 40 dias de gestação, pelas mesmas razões.

Uma condição que facilita o exame, consideravelmente, é o jejum de sólidos e líquidos por um período mínimo de 12 horas antes da realização o exame (BUCKRELL, 1988; ALVES et al 1991a).

GEARHART et al (1988) verificaram a eficiência do diagnóstico de gestação nas ovelhas atribuindo a percentagem de $89,94 \%$ nos dias 25 a 50 e de $100 \%$ nos dias 51 a 150, através de acesso inguinal. Com a utilização da ultra-sonografia pela via retal as probabilidades de acerto foram de apenas $12,28 \%$ nos dias 0 - 25 e de $64,76 \%$ entre os dias 26 e 50. Neste sentido ALVES et al (1991b) obtiveram 96\% de acertos no diagnóstico, confrontados com a parição após o exame de 1226 ovelhas, com transdutor de $2,4 \mathrm{MHz}$, a partir dos 30 dias de gestação pela via inguinal.

DAVEY (1986) observaram que o tempo requerido para 0 diagnóstico depende do número de ovelhas prenhes a ser encontrado no lote a ser examinado. As gestações simples podem ser detectadas em 10 segundos, mas quando há suspeita de gêmeos há necessidade de 20 a 30 segundos adicionais, para confirmação. As vazias requerem um tempo mínimo de 20 a 30 segundos de investigação para 0 diagnóstico. Deste modo, o número de ovelhas possível de ser examinado, em média é de 150 por hora, ou 1200 por dia, dependendo principalmente da atuação dos auxiliares e da estrutura do estabelecimento no sentido de apresentar os animais para exame.

\section{CONCLUSÕES}

O diagnóstico gestacional, através da ecografia, apresenta nítidas vantagens sobre os outros métodos de detecção, permitindo ao médico veterinărio maior segurança no controle reprodutivo das fêmeas, e contribuindo para o aumento da produtividade.

\section{REFERÊNCIAS BIBLIOGRÁFICAS}

ALVES, L.C., NEVES, J.P., LUZ, S. 
L.N. Aspectos do diagnóstico de gestação por ultra-sonografia na ovelha. In: CONGRESSO BRASILEIRO DE REPRODUÇÃO ANIMAL, 1991. Belo Horizonte, MG. Resumos ... Belo Horizonte, 1991a, 470 p. p. 397.

ALVES, L.C., NEVES, J.P., LUZ, S. L.N. Avaliação da ultra-sonografia abdominal para o diagnóstico de gestação em ovelhas. In: CONGRESSO BRASILEIRO DE REPRODUÇÃO ANIMAL, 1991. BelO Horizonte, MG. Resumos ... Belo Horizonte, $1991 \mathrm{~b}, 470$ p. p. 398.

BOYD, J.S., OMRAN, S.N., AYLUFFE, T.R. Use pf a high frequency transducer with real time B-mode ultrasound scanning to identify early pregnancy in cows. Veterinary Record, $v$. $123, p .8-11,1988$.

BUCKREL, B.C. Applications of ultrasonography in reproduction in sheep and goats. Theriogenology, v. $20, \mathrm{n} .1$, p. 71-84, 1988 .

CHAFFAUX, St., BIANCHI, P., BHAT, P., et al. L"ecografie en temps réel par voie transrectaleInterêt pour le diagnostic de gestation chez la vache. Recueil de Médecine Vetérinaire, v. 164, n. 2, p. 101-108, 1988 .

CHEVALIER, F., PALMER, E. Ultrasound echography in the mare. Journal of Reproduction and Fertility, n. 32, p. 423-430, 1982 .

CORTE, F.D. A ecografia no diagnóstico ginecológico eqüino. Santa Maria, 1990. 77 p. Tese (Mestrado em Medicina Veterinária) Curso de Pós-Graduação em
Medicina Veterinária. Universidade Federal de Santa Maria, 1990 .

CURRAN, S., PIERSON, R.A., GINTHER, O.J. Ultrasonographic appearance of the bovine conceptus from days 10 throught 20. Journal the American Veterinary Medical Association, v. 10, p. 1289-1294, 1986a.

CURRAN, S., PIERSON, R.A., GINTHER, O.J. Ultrasonographic appearance of the bovine conceptus from days 20 trought 60. Journal the American Veterinary Medical Association, v. 10, p. 1295-1302, 1986b.

DAVEY, C.G. An evaluation of pregnancy testing in sheep using a real-time ultrasound scanner. Australian Veterinary Journal, v. 63, n. 10, p. 347-348, 1986.

FISSORE, R.A., EDMONDSON, A.J., PASHEN, R.L. et al. The use of ultrasonography for the study of the bovine reproductive tract. II. Non-pregnant, pregnant and pathological conditions of the uterus. Animal Reproduction Science, $\mathrm{n}$. 12 , p. 167-177, 1986.

GEARHART, M.A., WINGF IELD, W.E. , KNIGHT, A.P. et al. Real-Time ultrasonography for determining pregnancy status and viable numbers in ewes. Theriogenology, v. $30, \mathrm{n} .2$, p. $323-337,1988$.

GINTER, O.J. Mobility of the early equine conceptus. Theriogenology, v. 19, n. 4, p. 603-611, 1983 .

GINTHER, O.J. Ultrasonic imaging and reproductive events in the mare. Wisconsin: 
Madison, $1986,378 \mathrm{p}$.

GRIFFITH, C.R. Basic physics. In: SABBAGHA, R.E. Diagnostic ultrasound applied to obstetrics and gynecology. Maryland: Harper \& Row, 1980, cap. 1, p. $1-10$.

GRUNERT, E ., BERCHTOLD, M. Fertilitätsstörungen beim weyblichen Rind. Berlin: Verlag Paul Parey, 1982, 522 p.

HAFEZ, E.S.E. Reproduction in Farm Animals. 5 ed. Philadelphia: Lea \& Febiger, 1987, $649 \mathrm{p}$.

KASTELIC, J.P., CURRAN, S., GINTHER, O.J. ACcuracy of ultrasonography for pregnancy diagnosis on days 10 to 22 in heifers. Theriogenology, v. 31, n. 4, p. 813-820, 1989 .

KASTELIC, J.P., BERGFELT, D.R., GINTHER, O.J. Ultrasonic detection of the conceptus fluid on days 10 to 22 in heifers. Theriogenology, v. 35, n. 3, p. 569-581, 1991.

PALMER, E., DRIANCOURT, M.A. Use the ultrasound echography in equine gynocology. Theriogenology, v. 13, p. 203-216, 1980 .

PIERSON, R.A., GINTHER, O.J. Ultrasonic imaging of the ovaries and uterus in cattle. Theriogenology, v. $29, \mathrm{n} .1, \mathrm{p}$. $21-37,1988$.

PIETERSE, M.C., SZENCI, O., WILLEMSE, A.H. et al.i Early pregnancy diagnosis in cattle by means of linear-array realtime ultrasound scanning of the uterus and a qualitative and quantitative milk progesterone test. Theriogenology, v. 33, n. 3, p. 697-707, 1990 .
PIPERS, F.S., ZENT, W., HOLDER, $R$. et al. A ultrasonography as an adjunct to pregnancy assesments in the mare. Journal the American Veterinary Medical Association, n. 184 , p. $328-334,1984$.

SERGEEV, L., KLEEMANN, D.O., WALKER, S. et al. Real-time ultrasound imaging for predicting ovine fetal age. Theriogenology, v. $34, \mathrm{n} .3, \mathrm{p}$. 593-601, 1990.

SIMPSON, D.J., GREENWOOD, R.E.S., RICKETS, S.W. et al. Use of ultrasound echography for early diagnosis of a single and twin pregnancy in the mare. Journal of Reproduction and Fertility, n. 32 , p. 431-439, 1982 .

TOTEY, S.M., SINGH, G., TANEJA, M., et al. Ultrasonography for detection of early pregnancy following embryo transfer in unknown breed of Bos indicus cows. Theriogenology, v. 35, n. 3, p. 487-497, 1991 .

WATT, B.R., ANDERSON, G.A., CAMPBELL, I.P. A comparison of six methods used for detecting pregnancy in sheep. Australian Veterinary Journal, $v .61, \mathrm{n}$. 12 , p. 377-382, 1984.

WHITE, I.R., RUSSEL, A.J.F. Determination of foetal numbers in sheep by real-time ultrasonic scanning. In: BODEN, E. Sheep and Goat Practice. London: Baillière Tindall, 1991. Cap. 4, p. 41-50.

WILKINS, J.F., FOWLER, D.G., BINDON, B.M., et al. Measuring foetal loss with real time scanning. Animal Production in Australia, v. 15, p. 768, 1984. 